\title{
Isolated Foetal Ascites: A Case Report
}

\author{
Ilker Arikan, Aykut Barut ${ }^{\mathrm{a}, \mathrm{c}}$, Muge Harma ${ }^{\mathrm{a}}$, Mehmet Ibrahim Harma ${ }^{\mathrm{a}}$, Suat Dogan ${ }^{\mathrm{b}}$
}

\begin{abstract}
When foetal ascites is found alone (i.e., without other signs of hydrops), it may represent a separate problem requiring a different management strategy, leading to a different outcome. A 24-year-old primigravida was referred to our institution at 27 weeks of gestation with a diagnosis of isolated foetal ascites. No other pathology was detected on ultrasonography or a laboratory examination. The patient delivered a male infant weighing $3020 \mathrm{~g}$ at 38 weeks of gestation with Apgar scores of 7 and 9 at 1 and 5 minutes, respectively. The newborn was operated on by a paediatric surgery team with an indication of intrauterine extra-hepatic bile duct perforation in the first post-partum week, and was subsequently discharged from the hospital without any complications. Isolated foetal ascites is a rare and separate situation from foetal hydrops. The perinatal outcome for isolated ascites is much better than that for hydrops foetalis.
\end{abstract}

Keywords: Intrauterine bile duct perforation; Isolated foetal ascites

\section{Introduction}

Foetal ascites is most often seen in association with foetal

Manuscript accepted for publication December 14, 2011

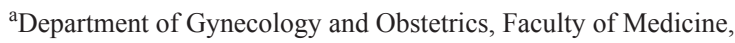
Zonguldak Karaelmas University, Zonguldak, Turkey

${ }^{b}$ Obstetrics and Gynecology Clinic, Van-Baskale State Hospital, Baskale, Van, Turkey

${ }^{\mathrm{c} C o r r e s p o n d i n g ~ a u t h o r: ~ A y k u t ~ B a r u t, ~ Z o n g u l d a k ~ K a r a e l m a s ~ U n i v e r s i t y, ~}$ Faculty of Medicine, Department of Gynecology and Obstetrics, Kozlu, 67600, Zonguldak, Turkey. Email: aykbar@yahoo.com

doi: $10.4021 / \mathrm{jmc} 475 \mathrm{w}$ hydrops or as one of the early manifestations of hydropic decompensation. When ascites is first identified, it is important to determine whether it is isolated or whether other signs of hydrops are present, including skin oedema, scalp oedema, pleural effusions, pericardial effusion, and tricuspid regurgitation. The prognosis and therapy for a foetus with hydrops depends on the aetiology. However, when foetal ascites is seen alone (i.e., without other signs of hydrops), it may represent a separate problem requiring a different management strategy, leading to a different outcome [1].

A careful search for underlying causes should be undertaken in all cases of apparently isolated ascites. A systematic protocol for the diagnostic work up of foetal ascites should be followed, as an aetiology or associated disorder can be identified in $92 \%$ of cases $[2,3]$.

Numerous mechanisms have been implicated in the generation of ascites, including abnormal lymphatic drainage; obstruction of venous return, as observed for any spaceoccupying lesion in the thorax; cardiac failure; decreased plasma oncotic pressure, as in foetal anaemia; hepatic insufficiency (storage disease) or congenital nephrosis; increased capillary permeability; urinary tract obstruction; or meconial peritonitis [4].

Infections such as congenital syphilis, cytomegalovirus (CMV), varicella, toxoplasmosis, and hepatitis A can uncommonly cause foetal ascites [1].

Here, we present a case of prenatally diagnosed isolated foetal ascites secondary to perforation of the extra-hepatic bile duct.

\section{Case Report}

A 24-year-old primigravida was referred to our institution at 27 weeks gestation with a diagnosis of isolated foetal ascites. Further work-up revealed a normal foetal echocardiogram scan and a normal karyotype on amniocentesis. The patient has an A-Rh positive blood type and was rubella immune. Tests for hepatitis B and C, CMV, toxoplasmosis, herpes simplex, and syphilis were all negative.

Our initial ultrasound at 27 weeks of gestation showed severe isolated foetal ascites and peritoneal calcification (Fig. 


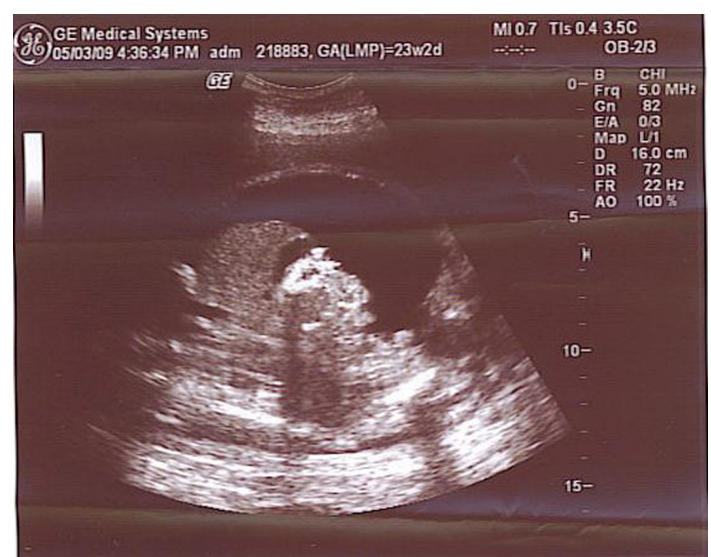

Figure 1. Severe isolated foetal ascites.

1) without any other structural anomalies. Two weeks later, we performed a foetal paracentesis, and a laboratory workup revealed sterile peritonitis. Cultures of the ascitic fluid for TORCH (toxoplasmosis, other agents, rubella, CMV, herpes simplex) infections were all negative. The foetal ascites disappeared on a subsequent ultrasound examination performed at 33 weeks of gestation (Fig. 2).

The patient presented to labour and delivery at 38 weeks of gestation with premature rupture of the membranes and subsequently delivered a male infant weighing $3020 \mathrm{~g}$ with Apgar scores of 7 and 9 at 1 and 5 minutes, respectively. The indication for caesarean delivery was a foetal breech malpresentation.

Subsequently, the newborn was operated on by a paediatric surgeon with the indication of an intrauterine extrahepatic bile duct perforation in the first post-partum week and was discharged from the hospital without any problems.

\section{Discussion}

Isolated ascites is diagnosed by demonstrating fluid surrounding the liver, spleen, bowel, extra-hepatic portion of the umbilical vein, falciform ligament, or greater omentum. When discovered on an initial sonogram, ascites should be followed by an ultrasound approximately 1 week later to determine whether progression to foetal hydrops has occurred. If the ascites remains isolated to the foetal abdomen, then progression to hydrops is much less likely [1].

Isolated ascites is most often secondary to an intra-abdominal disorder, rather than a generalized condition. It is most often secondary to obstructed uropathy; however, $20 \%$ of cases are due to gastrointestinal disorders [4-8]. Of these, meconium peritonitis is the most common cause, which results from a bowel obstruction [5].

The work-up for any case of ascites should include a detailed ultrasound examination to exclude the presence of any associated foetal abnormalities. The mother should be

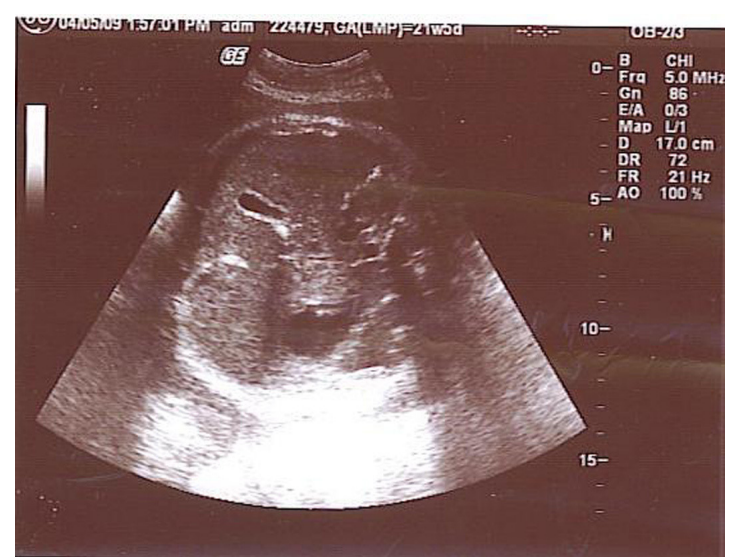

Figure 2. Disappearance of foetal ascites.

screened for the presence of viral infections such as parvovirus, CMV, hepatitis, varicella, herpes simplex, rubella, syphilis, and toxoplasmosis [1,2]. Additionally, the blood rhesus factor and blood antibody titres of the mother should be identified.

An amniocentesis for foetal karyotyping should be considered, as the incidence of chromosomal abnormalities could be up to $15 \%$ [9]. Other than a cytogenetic analysis, evaluating for a possible foetal infection (TORCH titres, antigen-specific IgM/IgG) and a prenatal diagnosis of inherited metabolic diseases are helpful $[1,2]$. These data could also be obtained from foetal blood sampling.

A foetal paracentesis can be performed to evaluate the meconium, protein/lymphocyte counts, TORCH titres, and the foetal karyotype [1]. Massive compression of the chest caused by intra-abdominal ascites before 24 weeks of gestation may lead to pulmonary hypoplasia, but it is uncertain whether foetal paracentesis is helpful in this situation $[1,10]$. A foetal echocardiogram is also warranted to rule out a cardiac anomaly or arrhythmia [1].

Bishry reported a series of twelve cases with isolated foetal ascites without any other anomalies detected antenatally, and ten survived postnatally. Only one of the ten cases had ileal atresia detected postpartum, which was repaired. The other nine cases had no abnormalities that could be detected either antenatally or postnatally. The two cases of foetal loss were diagnosed before 20 weeks of gestation and one had laryngeal atresia, which was terminal [2].

A larger series reported by Favre et al. detected approximately the same survival rate $(8 / 8)$ for idiopathic isolated foetal ascites, in which no cause could be demonstrated during the foetal and neonatal periods [4]. They documented a significant relationship between survival rate and gestational age at diagnosis.

Furthermore, Bishry could detect the cause of isolated foetal ascites in 26 of 28 cases antenatally [2], whereas Favre et al. could detect the cause in only 8 of 25 cases [4].

In our case, we could not identify any cause using data 
from the work-up we performed antenatally.

\section{Conclusion}

Isolated foetal ascites is a rare and separate condition from foetal hydrops. The perinatal outcome for isolated ascites is much better than that for hydrops foetalis. An extensive work-up should be conducted to demonstrate the cause, since most cases are associated with other abnormalities.

\section{References}

1. Ulreich S, Gruslin A, Nodell CG, Pretorius HD. Fetal Hydrops and Ascites. In: Nyberg DA, McGahan JP, Pretorius DH, Pilu G, editors. Diagnostic imaging of fetal anomalies. New York: Lippincott Williams and Wilkins; 2003. pp 713-745.

2. El Bishry G. The outcome of isolated fetal ascites. Eur J Obstet Gynecol Reprod Biol. 2008;137(1):43-46.

3. Schmider A, Henrich W, Reles A, Kjos S, Dudenhausen JW. Etiology and prognosis of fetal ascites. Fetal Diagn Ther. 2003;18(4):230-236.

4. Favre R, Dreux S, Dommergues M, Dumez Y, Luton D,
Oury JF, Fiblec BL, et al. Nonimmune fetal ascites: a series of 79 cases. Am J Obstet Gynecol. 2004;190(2):407412.

5. Agrawala G, Predanic M, Perni SC, Chasen ST. Isolated fetal ascites caused by bowel perforation due to colonic atresia. J Matern Fetal Neonatal Med. 2005;17(4):291294.

6. Ohno Y, Koyama N, Tsuda M, Arii Y. Antenatal ultrasonographic appearance of a cloacal anomaly. Obstet Gynecol. 2000;95(6 Pt 2):1013-1015.

7. Persutte WH, Lenke RL, Kropp KA. Atypical perentation of fetal obstructive uropathy. J Diagn Med Sonogr 1989;1:12-15.

8. Mahony BS, Callen PW, Filly RA. Fetal urethral obstruction: US evaluation. Radiology. 1985;157(1):221224.

9. Jauniaux E, Van Maldergem L, De Munter C, Moscoso G, Gillerot Y. Nonimmune hydrops fetalis associated with genetic abnormalities. Obstet Gynecol. 1990;75(3 Pt 2):568-572.

10. Benacerraf BR, Frigoletto FD, Jr., Wilson M. Successful midtrimester thoracentesis with analysis of the lymphocyte population in the pleural effusion. Am J Obstet Gynecol. 1986;155(2):398-399. 